BULL. AUSTRAL. MATH. SOC.

VOL. 9 (1973), 137-140.

\title{
A final remark on extending to strict total orders in modules
}

\author{
Isidore Fleischer
}

\begin{abstract}
The remark is that the recent work in this Bulzetin dealing with the extension of a partial to a total strict order on a module over a partially ordered ring falls under standard order extension results for operator groups once it is noted that the added requirement of strictness just comes to injective operation of the positive scalars. These standard results are in turn generalized to a universal algebra setting.
\end{abstract}

Included in the Corrigenda [5] to [4] should have been a retraction of the claim to generalize [1], p. 113, Theorem 1: in fact, that theorem deals with rings and not, as claimed in [4], with abelian groups. To be sure, a footnote on $p .113$ of [1] refers back to the analogous theorem for groups on p. 34 , but it turns out to be formulated for not necessarily commutative ones. Indeed, according to a remark on p. 37 this version is valid even for operator groups, which makes it available for modules rather than the other way round. Of course, the formulation is for arbitrary rather than for strict orders: however, if a module admits any strict total order the positive scalars necessarily operate injectively; conversely, injective operation of the positives entails the strictness of every partial order. This observation permits dispensing with the major part of [4] and [6] in favor of known results for abelian operator groups: for example, to obtain the main theorem of [4] it suffices merely to note that Condition 2) for $n=1$ yields the injectivity of positive scalars; or Corollary 3 of [6] can be strengthened to apply to every partial order

Received 28 March 1973. 
on a torsionfree module, rather than to just the trivial one, and under a broader sense of torsionfree, by appeal to [1], p. 40, Corollary 14.

It might be worth while to point out that all of the order extension theorems are instances of a general result which can be extracted from an early and unjustly neglected article [3]. The focus of [3] is conjunction of quasi-orders rather than strengthening of orders, so that a small amount of reformulation is needed to bring the argument to bear also on this situation. (On the other hand, [3] has a much broader scope largely exceeding the pure order context.)

$A$ is a set; 2 (one should think of quasi-orders) a set of binary relations on $A$, including the all relation and forming a complete lattice under inclusion; $P$ (for example, the partial orders) a subset of 2 including with any relation all those in $Q$ smaller than it and with any ascending chain its supremum in 2 . A relation is total if for every pair $a, a^{\prime} \in A$, it contains either $\left(a, a^{\prime}\right)$ or $\left(a^{\prime}, a\right)=-\left(a, a^{\prime}\right)$.

THEOREM. A necessary and sufficient condition for $R$ to be contained in a total relation of $P$, is that for every finitely many pairs $\alpha_{1}, \ldots, \alpha_{n} \in A \times A$, there exist an assignment $\varepsilon_{1}, \ldots, \varepsilon_{n}$ of signs + or - such that $R\left[\varepsilon_{1} \alpha_{1}, \ldots, \varepsilon_{n} \alpha_{n}\right]$, the smallest relation in $Q$ containing $R$ and all the $\varepsilon_{i} \alpha_{i}$, actually belongs to $P$.

For the proof, observe that if $R^{*}$ is the smallest relation in 2 containing a family $R_{i}$, then $R^{*}\left[\alpha_{1}, \ldots, \alpha_{n}\right]$ is the smallest containing the family $R_{i}\left[\alpha_{1}, \ldots, \alpha_{n}\right]$. It follows readily that the set of $R^{\prime} s$ satisfying the stated condition is closed under the taking of suprema in 2 of its chains. But a relation maximal in the set is total: indeed, with every $R$ satisfying the condition, so does at least one of $R[\alpha], R[-\alpha]$ for every pair $\alpha$ : else by combining with each other and $\alpha$ the finite sets for which no sign assignment can be realized in $P$ compatibly with $R[\alpha]$ and $R[-\alpha]$, there would result a finite set having no sign assigmment compatible with $R$ in $P$. Inasmuch as necessity follows directly from the definitions, this completes the proof.

To obtain from this a characterization of the relations which can be extended to be antisymmetric and total in 2 , or of the confunction of the 
total ones containing a given relation, one will want to have unions of chains in $Q$ formed set theoretically: its anti-symetric members, or those not containing a given $\alpha$, can then function as $P$. The condition for $R$ to be contained in a total anti-symmetric member of 2 will read: for every $\alpha_{1}, \ldots, \alpha_{n}$ there exist $\varepsilon_{1}, \ldots, \varepsilon_{n}$ with $R\left[\varepsilon_{1} \alpha_{1}, \ldots, \varepsilon_{n} \alpha_{n}\right]$ anti-symmetric; while the $\alpha$ in the conjunction of the total members containing $R$ - that is, which cannot be excluded by some total member containing $R$ - will be just those for which there exist $\alpha_{1}, \ldots, \alpha_{n}$ such that $\alpha$ belongs to $R\left[\varepsilon_{1} \alpha_{1}, \ldots, \varepsilon_{n} \alpha_{n}\right]$ for each of the $2^{n}$ possible sign assignments.

As for 2 , it consists habitually of the quasi-orders compatible with an algebraic structure on $A$. According to [2], this compatibility should consist in the algebraic operations becoming isotone (or antitone) self-maps of $A$ on replacing all but one of their arguments by elements from selected (preferably by some elementary condition involving the order) subsets of $A$. This is conceived for a structure defined by internal operations exclusively; but by construing these self-maps as operators on $A$, order preservation by external scalars will also be captured by the formalism.

Thus let $\Omega$ be a supplementary set operating on $A$ and suppose every quasi-order $R$ on $A$ assigned a subset $\Omega(R)$ of $\Omega$ increasing with increasing $R$ - this makes the set 2 of those quasi-orders $R$ for which the operators in $\Omega(R)$ act isotonely a complete lattice: in fact, even closed under set theoretic intersection. To have it also closed under set theoretic chain union, it suffices to have the unions of chains of relations assigned the unions of the corresponding chains of subsets of $\Omega$.

For example for groups $\Omega(R)$ is constant, consisting of the translations along with any external operators which one might require to preserve order, such as the positive scalars for modules; while for rings it must also include the multiplications by those ring elements which become positive for $R$. 


\section{References}

[1] L. Fuchs, Partially ordered algebraic systems (Pergamon, Oxford, London, New York, Paris, 1963).

[2] L. Fuchs, "On partially ordered algebras I", Colloq. Math. 14 (1966), 115-130.

[3] Paul Lorenzen, "Teilbarkeitstheorie in Bereichen", Math. 2. 55 (1952), 269-275.

[4] P. Ribenboim, "On the extension of orders in ordered modules", BuZl. Austral. Math. Soc. 2 (1970), 81-88.

[5] P. Ribenboim, "On the extension of orders in ordered modules: Corrigenda", Buzl. Austral. Math. Soc. 4 (1971), 288.

[6] D. Spoerel, "A remark on Ribenboim's paper 'On the extension of orders in ordered modules" ", Bull. Austral. Math. Soc. 6 (1972), 251-253.

Département de Mathématiques,

Université de Montréal,

Montréal,

Canada. 